\title{
Relationships between exercise capacity and anxiety, depression, and cognition in patients with heart failure
}

Oronzo Chiala, Ercole Vellone, Leonie Klompstra, Giorgio Alberto Ortali, Anna Strömberg and Tiny J aarsma

The self-archived postprint version of this journal article is available at Linköping University Institutional Repository (DiVA):

http:// urn.kb.se/ resolve?urn=urn:nbn:se:liu:diva-153190

N.B.: When citing this work, cite the original publication.

Chiala, O., Vellone, E., Klompstra, L., Ortali, G. A., Strömberg, A., J aarsma, T., (2018), Relationships between exercise capacity and anxiety, depression, and cognition in patients with heart failure, Heart \&Lung, 47(5), 465-470. https:// doi.org/ 10.1016/j.hrtlng.2018.07.010

Original publication available at:

https:// doi.org/ 10.1016/j.hrtlng.2018.07.010

Copyright: Elsevier (12 months)

http:// www.elsevier.com/

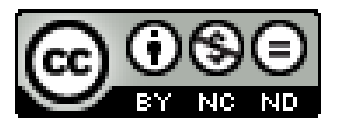




\section{Relationships between exercise capacity and anxiety, depression, and cognition in patients with heart failure}

\section{ABSTRACT \\ Background}

Symptoms of anxiety, depression, and cognitive impairment are common in heart failure (HF) patients, but there are inconsistencies in the literature regarding their relationship and effects on exercise capacity.

\section{Objectives}

The aim of this study was to explore the relationships between exercise capacity and anxiety, depression, and cognition in HF patients.

\section{Methods}

This was a secondary analysis on the baseline data of the Italian subsample (n=96) of HF patients enrolled in the HF-Wii study. Data was collected with the 6-minute walk test (6MWT), Hospital Anxiety and Depression Scale, and Montreal Cognitive Assessment.

\section{Results}

The HF patients walked an average of 222 (SD 114) meters on the 6MWT. Patients exhibited clinically elevated anxiety (48\%), depression (49\%), and severe cognitive impairment (48\%). Depression was independently associated with the distance walked on the 6MWT.

\section{Conclusions}

The results of this study reinforced the role of depression in relation to exercise capacity and call for considering strategies to reduce depressive symptoms to improve outcomes of HF patients. 
Keywords: heart failure, exercise capacity, anxiety, depression, cognitive impairment

Declaration of interests: The authors have nothing to declare.

\section{ABBREVIATION LIST}

6MWT: 6-minute walk test

BMI: body mass index

CBT: cognitive behavioral therapy

HADS: Hospital Anxiety and Depression Scale

HF: heart failure

ICBT: internet-based cognitive behavioral therapy

MoCA: Montreal Cognitive Assessment

NYHA: New York Heart Association

QOL: quality of life

SD: standard deviation

SPSS: Statistical Package for the Social Sciences

VIF: variance inflation factor 


\section{AKNOWLEDGEMENTS}

The authors would like to thank Roberto Corsi, the Health Director of Casa di Cura Villa delle Querce in Nemi (Rome), Italy for allowing this research and providing support for the logistical issues. They would also like to give special thanks to Physical Therapist Manlio Bitocchi for helping during the recruitment phase.

\section{FUNDING}

The HF-Wii study was supported by the Swedish National Science Council (K2013-69X22302-01-3), The Swedish Heart and Lung Association (E085/12 and E120/15), The Swedish Heart-Lung Foundation (20130340), the Vårdal Foundation (2014-0018), the Medical Research Council of Southeast Sweden (FORSS 474681), and the Swedish Research Council for Health, Working Life, and Welfare (VR-FORTE) 2014-4100. 


\section{INTRODUCTION}

Heart failure (HF) is a chronic syndrome affecting $1-2 \%$ of the European population. ${ }^{1}$ In US, 6.5 million Americans have HF, with over 1 million new cases diagnosed annually. ${ }^{2}$ HF has been increasing in prevalence, which can be explained, in large part, by population ageing and improvements in cardiac disease treatment. In Europe, when considering all hospital admissions, 0.32-3.73\% are due to HF. ${ }^{1}$ Moreover, HF is associated with high morbidity and mortality, as well as considerable healthcare costs. ${ }^{3}$

Psychological and cognitive problems are common in patients with HF and affect their quality of life (QOL). ${ }^{4}$ Approximately $60 \%$ of HF patients have anxiety, 30\% have depression, and $40 \%$ have cognitive impairments, as found in several studies using psychometrically sound instruments to measure these variables. ${ }^{5-7}$ Due to their limited cardiac function and symptoms, HF patients are known to have a reduced exercise tolerance. ${ }^{3}$

The exercise capacity is defined as the maximum amount of physical exertion that a person can sustain. ${ }^{8}$ An exercise intolerance is common in HF patients, and it can lead to reductions in their skeletal muscle mass and in physical activity adherence. ${ }^{9}$ Moreover, a reduced exercise capacity in HF patients is associated with worse outcomes, such as a higher mortality, higher number of HF-specific hospitalizations, and lower QOL. ${ }^{3,10}$ Despite the positive effects of exercise training on outcomes, only about $30 \%$ of the HF patients adhere to their exercise recommendations. ${ }^{11}$

Several factors are related to the exercise capacity of HF patients, ${ }^{12,13}$ including symptoms of anxiety, depression, and cognitive impairment ${ }^{12,14-18}$; however, their roles remain unclear, and the study results are inconsistent. With regard to anxiety, some studies have found that a higher anxiety level is associated with a lower exercise capacity in HF patients, ${ }^{17}$ but other studies have not confirmed this. ${ }^{16,18}$ Some studies reported a modest correlation between depressive symptoms 
and exercise capacity, ${ }^{18-20}$ but others did not. ${ }^{21}$ Regarding cognitive impairment, Baldasseroni et al. ${ }^{12}$ found a correlation between cognitive function and the exercise capacity of HF patients, while Alosco et al. found that this correlation was significant only with the areas of executive functions and language..$^{22}$

It is also known that anxiety, depression, and cognitive impairment can coexist in HF patients, ${ }^{23,24}$ and that they can influence each other. ${ }^{24}$ The relationship between exercise capacity and these variables is not unidirectional. For example, on one hand a lack of exercise might trigger or strengthen depressive symptoms and on the other hand depression may lead to a sedentary lifestyle, with consequent lack in motivation to engage in exercise training, resulting in a consequent reduction of exercise capacity. ${ }^{20}$ To date, these three variables have been investigated most often individually, ${ }^{16-18,21,25}$ without identifying the independent predictor of the exercise capacity among them. Knowledge of the independent factors related to the exercise capacity could help to design future tailored interventions to improve exercise, with a potential effect on mortality, HF-specific rehospitalization, and QOL. Therefore, the aim of this study was to evaluate the relationship between symptoms of anxiety, depression and cognition and the exercise capacity of HF patients.

\section{METHODS}

\section{Participants and setting}

The patients enrolled in the present study is the Italian subsample of the HF-Wii study, ${ }^{25}$ an international randomized controlled trial aimed to improve the exercise capacity of HF patients with exergaming (clinicaltrial.gov, identifier: NCT01785121). The patients were recruited at the Villa delle Querce rehabilitation hospital in Nemi (Rome), Italy from October 2014 through December 2016. The eligibility criteria were as follows: (a) a diagnosis of HF 
[New York Heart Association (NYHA) I-IV], independent from the ejection fraction; (b) older than 18 years, without an upper age limit; and (c) able to speak/understand the Italian language. The exclusion criteria were as follows: (a) unable to use the Wii exergame due to visual, hearingcognitive, or motor impairments, as judged by the research team; (b) unable to fill in data collection material; (c) a life expectancy shorter than 6 months; and (d) already having a Wii exergame.

\section{Ethical considerations}

This study was conducted in accordance with the principles of the Declaration of Helsinki (version 2008) and according to the Medical Research Involving Human Patients Act of the countries involved in this multicenter study. In Italy, ethical approval was obtained (n. 101.14 prot. N. 47867 of 02.07 .2014 ) before the study began.

\section{Data collection process}

Data collection was conducted in five steps. First, a general screening of eligible patients was made, considering the inclusion criteria. Second, the exclusion criteria were evaluated. Third, if the criteria were met, the HF-Wii study was introduced according to its protocol ${ }^{25}$ and the informed consent was signed. Then, the questionnaires were administered to patients. Finally, the patients performed the 6MWT and further medical data were collected from the patients medical file.

\section{Measurements}

The exercise capacity was measured using the 6-minute walk test (6MWT), which measures the distance walked (in meters) on a linear track marked with visible signs on a flat and 
firm surface. It is a frequently used, reliable, and well-validated measure of exercise capacity in patients with HF. ${ }^{26}$ For this paper, a cut-off of 300 meters was used according to Rostagno et al., ${ }^{27}$ who reported a higher mortality in the HF patients who covered less than 300 meters during the 6MWT.

Anxiety and depression were measured with the Hospital Anxiety and Depression Scale (HADS $),{ }^{28}$ which is a 14 -item self-reported questionnaire incorporating the anxiety and depression subscales. Each item is scored from 0 to 3, with a score from 0 to 21 for each subscale. Higher scores mean higher anxiety and depression levels, with a score above 7 indicating a clinical state of anxiety and depression. ${ }^{29}$ The HADS is valid and reliable for measuring anxiety and depression in HF patients. ${ }^{30}$ In this study, the Cronbach's alphas were 0.79 and 0.67 for the anxiety and depression subscales, respectively.

Cognitive impairment was evaluated using the Montreal Cognitive Assessment (MoCA), ${ }^{31}$ which assesses different cognitive domains, such as attention and concentration, executive functions, memory, language, visuoconstructional skills, conceptual thinking, calculations, and orientation. The total possible score ranged from 0 to 30 , with a high score indicating better cognition. As reported in previous studies, the patients were classified as "no to light cognitive impairment” with a score from 27 to 30, “mild cognitive impairment” with a score from 18 to 26, “moderate cognitive impairment” with a score from 10 to 17, and "severe cognitive impairment” with a score lower than $10 .^{31,32}$ The MoCA is a valid and reliable tool for the HF population. ${ }^{33}$ In this study, the Cronbach’s alpha was 0.75.

Information about the age, gender, education, marital status, smoking, time sincediagnosis, and exercise during the last week was collected using a questionnaire. The data on HF etiology, ECG rhythm, ejection fraction, NYHA functional class, body mass index (BMI), and type of medications prescribed were collected from the patients' medical records. 


\section{Statistical analysis}

Since this was a secondary analysis of baseline data, no power analysis was performed; the sample size was based on the Italian subsample of patients defined by the HF-Wii trial.

The statistical data analysis was performed in four steps. First, descriptive statistics (e.g. the mean and SD) were used to describe the sociodemographic and clinical characteristics of the participants, as well as the 6MWT, HADS, and MoCA scores. Normal distribution of the data was tested by the Kolmogorov-Smirnov test. Second, an analysis of variance was used to assess the differences in the meters walked during the 6MWT, and according to the fixed cut-offs for the HADS and MoCA. A Bonferroni post-hoc analysis was applied to evaluate the significant differences between three cognitive impairment groups as assessed by the MoCA (no to light cognitive impairment, mild, and moderate to severe). Third, Spearman's rho correlations was applied due to non-normal distribution of continuous variables and for categorical/dichotomous ones to identify multicollinearity and the variables considered to be covariates of the regression model (age, gender, education, NYHA class, and BMI). Fourth, a stepwise regression model was determined with the exercise capacity as a dependent variable. In this model, the predictors considered were the HADS and MoCA scores and all of the covariates that were significantly correlated with the 6MWT scores: age, gender (male vs. female), NYHA class (as continuous variable). The significance threshold was fixed at $\mathrm{p}<.05$. The Durbin-Watson test and the variance inflation factors (VIF) were calculated to assess the possible collinearity. A VIF of $>10$ and a Durbin-Watson test value $<1$ or $>3$ was regarded as indicative of autocorrelation.

All of the data were analyzed using IBM SPSS Statistics for Windows, Version 22.0 (IBM Corp., Armonk, NY, USA), and a p-value below 0.05 was considered to be statistically significant. 


\section{RESULTS}

\section{Sociodemographic and clinical characteristics of the sample}

A total of $328 \mathrm{HF}$ patients were screened for the study, 232 were eligible and 96 agreed to participate (mean age $72 \pm 10$ years). The self-reported reasons for non-participating were as follows: lived too far from the enrollment center $(n=73)$, did not have time to participate $(n=56)$, lived in nursing homes or in religious facilities that not allowed to use exergames $(n=4)$, reported having other diseases that impeded them to participate in the study $(n=3)$. The patients who declined to participate were older (76 vs. 72 years; $\mathrm{p}<.001$ ) and had a higher NYHA class $\left(\chi^{2}=14.619 ; \mathrm{p}=.002\right)$ than those enrolled in the study. Most of the participants were men (73\%) with a Primary or High School education level, and most of the patients were married (58\%) (Table 1). The predominant causes of HF were cardiomyopathy (63\%) and hypertension (57\%), and most of the patients were classified as NYHA II and III (92.7\% in total) (Table 1). Only 54\% of patients were prescribed taking a beta blocker, 28\% were prescribed an ACE inhibitor and 13\% an ARB. Most of patients were prescribed a diuretic (88\%), (Table 1).

\section{Exercise capacity, anxiety, depression, and cognition}

The patients walked an average of 222 meters (SD 114.26) on the 6MWT. In total, 20 patients stopped before reaching 6 minutes for different reasons, mainly due to fatigue. Overall, 26 patients walked more than 300 meters (27\%), while 70 walked less than 300 meters (73\%).

The mean anxiety score as measured by the HADS was 6.96 (SD 4.79), while the mean depression score was 7.37 (SD 4.33). Based on a HADS cut-off score of 7, anxiety was found in 46 patients (48\%), while depression was found in 47 patients (49\%). Thirty-five patients (36.5\%) had both anxiety and depression above the cut-off scores. 
There were no statistically significant differences between the patients with anxiety and those without clinically relevant anxiety in terms of the meters walked in the 6MWT. However, the clinically depressed HF patients walked less than those who were not depressed, with a statistically significant difference $(\mathrm{p}=.030)$.

The mean score of the MoCA was 21.29 (SD 4.86). Based on the MoCA cut-off score, 11 patients (11\%) were classified as no to light cognitive impairment, 67 (70\%) had mild cognitive impairment, 17 (18\%) had moderate cognitive impairment, and 1 (1\%) had severe cognitive impairment.

The differences in the meters walked in the 6MWT were significant between the groups identified by the MoCA cut-offs ( $\mathrm{p}=0.023$ ), with the non-cognitively impaired HF patients walking more than the patients with cognitive impairment. A significant difference was found between the patients with no to light cognitive impairment and those with mild cognitive impairment (310.27 vs. 213.14, respectively; $\mathrm{p}=0.025$ ). There was also a significant difference between the patients with no to light cognitive impairment and those with moderate and severe cognitive impairment (310.27 vs. 202.94, respectively; $\mathrm{p}=0.039$ ). However, there was no significant difference between the mildly impaired and moderate/severely impaired patients with HF (Figure 1).

\section{Relationships among the 6MWT, HADS, and MoCA}

The correlations among the 6MWT, HADS, MoCA, and the covariates are reported in Table 2. The 6MWT was negatively correlated with anxiety $(\mathrm{r}=-.231, \mathrm{p}=.023)$ and depression $(\mathrm{r}=-.325, \mathrm{p}=.001)$, but positively correlated with cognition $(\mathrm{r}=.244, \mathrm{p}=.017)$. There were also statistically significant relationships between the 6MWT and age $(r=-.451, \mathrm{p}<.001)$, gender $(r=-$ 
$.407, \mathrm{p}<.001)$, and NYHA classification ( $r=-.540, \mathrm{p}<.001)$. With regard to gender, the male HF patients walked significantly further than the female patients.

In the stepwise regression analysis, with the $6 \mathrm{MWT}$ as the dependent variable and the HADS, MoCA, age, gender, and NYHA class as independent variables, the significant predictors of the 6MWT were the NYHA class, age, gender, and depression (Table 3). Specifically, those who walked more meters in the 6MWT were those patients with a lower NYHA class who were younger males with less depression. This model explained the 47\% variance in the 6MWT (Table 3).

\section{DISCUSSION}

To our knowledge, this is the first study that evaluated, in a single analysis, the relationships between the exercise capacity and anxiety, depression, and cognitive impairment. It showed the independent role of depression in predicting the exercise capacity.

The exercise capacity measured with the 6MWT in our study was comparable to some previous studies of HF patients, ${ }^{15,16}$ while some studies reported better performances in the 6MWT in HF patients. The patients in the studies with longer distances covered in the 6MWT were, in general, younger and in a lower NYHA class. ${ }^{12,18,34-36}$ These differences were reflected in our study, in which we found an independent relationship between the exercise capacity and patients' age, gender, and NYHA. This illustrates the importance of taking the demographics and NYHA into account when advising patients about exercise, and being realistic in one's expectations from exercise interventions.

When compared with HF outpatients in prior studies, we found an even higher number of patients reporting anxiety and depression. ${ }^{17,37}$ This difference might have been due to the inhospital recruitment; this study included patients that could have "felt sicker" than the general 
population of HF patients. A similar prevalence was reported in the hospitalized HF patients in other studies. $^{38}$

The levels of anxiety measured with the HADS in this study were similar ${ }^{34,36}$ or slightly higher than those reported in previous studies. ${ }^{15,17,18}$ Anxiety and depression may reduce a patient's independence or their motivation to be active. ${ }^{39}$ Our study showed a significant correlation between the anxiety and physical capacity, confirming the results of previous findings. ${ }^{17}$ However, this relationship was not confirmed in the regression analysis.

In this study, depression reached a prevalence similar to that of other studies of hospitalized HF patients, ${ }^{21,35,40,41}$ but with higher HADS scores (depression domain). ${ }^{15,17,18,34}$ With regard to depression, we confirmed the previous findings, and found an independent strong correlation between depression and the physical capacity. ${ }^{7,17-19,22,35,41}$

With regard to cognitive impairment, we found that a considerable number of patients (89\%) were at least mildly cognitively impaired, but almost 1 in 5 of the HF patients had moderate to severe cognitive impairment. This high prevalence of cognitive impairment can be explained in two ways. First, these results emerged in accordance with the MoCA cut-off values fixed in previous studies of HF patients, ${ }^{32}$ but recent meta-analyses have revealed lower cut-off scores to better identify patients with mild cognitive impairment. ${ }^{42,43}$ For example, following the cut-off score proposed by Carson et al., ${ }^{42}$ only $54 \%$ of the patients in this study had cognitive impairment. Second, the patients included in this study had suboptimal medication regimens that could have affected their cognitive function, ${ }^{44}$ as reported in a single studies of Italian HF patients. ${ }^{45}$ Similarly to the Cocchieri et al. study, ${ }^{45}$ our patients reported a medication regimen

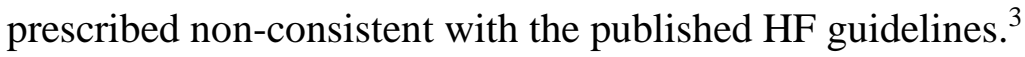

Moreover, we confirmed the relationship between cognitive impairment and the physical capacity according to other studies ${ }^{12,22}$; however, when correcting for other variables, this 
relationship was not statistically significant. This indicated that there clearly are variables other than cognition alone that could explain the lower exercise capacity in HF patients.

Although cognitive training strategies showed potential improvements in certain cognitive outcomes, and a consequent improvement in the exercise capacity in the 6MWT in a pilot study ${ }^{46}$ our results showed that the independent effect of depression should be taken into account when planning interventions for HF patients with cognitive problems. Our findings underscore the importance of including a psychological evaluation of depression, which may help develop more tailored interventions to improve the exercise capacity. The screening methods and effective interventions in depressive symptoms continue to be challenging topics, due to a considerable overlap between HF symptoms and depressive symptoms (i.e. weight loss, fatigue) ${ }^{47}$ Programs aimed specifically at reducing depression in HF patients, such as cognitive behavioral therapy ${ }^{48}$ or even internet-based cognitive behavioral therapy, ${ }^{49}$ or intervention that focus on cognition or other symptoms such as repeated recall ${ }^{50}$ or mindfullness ${ }^{51}$ may be promising in the future. We are aware that the relationship between physical activity and depression is a complex one, and it might not be unidirectional. ${ }^{48}$ However, targeting interventions at both reducing depression and improving physical activity might be needed to reduce the mortality and rehospitalization risks. ${ }^{19}$

One strength of this study was that, according to HF-Wii study protocol, we used wider recruitment criteria, without excluding patients for their age and cognitive limitation as assessed by cognitive test. This allowed us to have a more representative sampler. Interestingly, despite the good correlation between the cognition and exercise capacity, cognitive impairment was not a predictor of the meters walked in the 6MWT.

Among the limitations of this study, we should emphasize the use of in-hospital recruitment, which may have influenced the anxiety and depression levels due to the patients' 
possibly negative perceptions of their health status. Despite concerns about the effect of cognitive impairment on HADS, Stott et al. suggested how even patients with mild to moderate dementia can complete effectively the questionnaire. ${ }^{52}$ The small sample size was composed mainly of men with poor cognition and poor physical capacity, which may have limited the generalizability. Moreover, the explicit inclusion in a study focused on the exercise capacity could have influenced their physical performance. Finally, the use of a self-reported instrument, even if it was reliable and effective (HADS), to assess the anxiety and depression could have pushed the patients to select options that were socially desirable, exposing the results to a possible bias.

Even though strong correlations were found between the meters walked on the 6MWT and anxiety, depression, and cognitive impairment, the results of this study showed that depression, was the only predictor of the exercise capacity among them. In this study, other variables predicted exercise capacity (age, gender, NYHA class), but only the depression results, although challenging, more realistic to change. Anxiety, depression, and cognitive impairment may coexist in HF patients; therefore, a multidimensional approach is needed. When considering the strong relationship between depression and the exercise capacity, and the consequent effects on rehospitalization and mortality, it is important to focus attention on interventions to reduce depression in HF patients. Either traditional or internet-based cognitive behavioral therapy could represent a tailored strategy to improve HF patient outcomes. 


\section{REFERENCES}

1. Ponikowski SA, AlHabib KF, Cowie MR, Force TL, Hu S, Jaarsma T, Krum H, Rastogi V, Rohde LE, Samal UC, Shimokawa H, Siswanto BB, Sliwa K, Filippatos G. Heart failure: prevent disease and death worldwide. ESC Heart Failure. 2014;1:4-25.

2. Benjamin EJ, Virani SS, Callaway CW, et al. Heart Disease and Stroke Statistics-2018 Update: A Report From the American Heart Association. Circulation. 2018;137(12):e67-e492.

3. Ponikowski P, Voors AA, Anker SD, et al. 2016 ESC Guidelines for the diagnosis and treatment of acute and chronic heart failure: The Task Force for the diagnosis and treatment of acute and chronic heart failure of the European Society of Cardiology (ESC). Developed with the special contribution of the Heart Failure Association (HFA) of the ESC. European Journal of Heart Failure. 2016;18(8):891-975.

4. McMurray JJ. Clinical practice. Systolic heart failure. The New England journal of medicine. 2010;362(3):228-238.

5. Almeida OP, Beer C, Lautenschlager NT, Arnolda L, Alfonso H, Flicker L. Two-year course of cognitive function and mood in adults with congestive heart failure and coronary artery disease: the Heart-Mind Study. International psychogeriatrics / IPA. 2012;24(1):38-47.

6. Vellone E, Fida R, D'Agostino F, et al. Self-care confidence may be the key: A cross-sectional study on the association between cognition and self-care behaviors in adults with heart failure. International Journal of Nursing Studies. 2015;52(11):1705-1713.

7. Moser DK, Dracup K, Evangelista LS, et al. Comparison of prevalence of symptoms of depression, anxiety, and hostility in elderly patients with heart failure, myocardial infarction, and a coronary artery bypass graft. Heart \& lung : 2010;39(5):378-385.

8. Walker HKH, W.D.; Hurst, J.W. Clinical Methods: the history, physical and laboratory examinations - 3rd Edition. Boston: Butterworths; 1990.

9. Kinugawa S, Takada S, Matsushima S, Okita K, Tsutsui H. Skeletal Muscle Abnormalities in Heart Failure. International Heart Journal. 2015;56(5):475-484.

10. Palau P, Dominguez E, Nunez E, et al. Peak Exercise Oxygen Uptake Predicts Recurrent Admissions in Heart Failure With Preserved Ejection Fraction. Revista espanola de cardiologia. 2017.

11. Flynn KE, Piña IL, Whellan DJ, et al. Effects of exercise training on health status in patients with chronic heart failure: HF-ACTION randomized controlled trial. JAMA. 2009;301(14):1451-1459.

12. Baldasseroni S, Mossello E, Romboli B, et al. Relationship between cognitive function and 6minute walking test in older outpatients with chronic heart failure. Aging Clinical and Experimental Research. 2010;22(4):308-313.

13. Ahmeti $A$, Henein $M Y$, Ibrahimi $P$, et al. Quality of life questionnaire predicts poor exercise capacity only in HFpEF and not in HFrEF. BMC Cardiovascular Disorders. 2017;17(1):268.

14. Kim J, Shin MS, Hwang SY, et al. Memory loss and decreased executive function are associated with limited functional capacity in patients with heart failure compared to patients with other medical conditions. Heart \& Lung. 2017.

15. Witham MD, Argo IS, Johnston DW, Struthers AD, McMurdo ME. Predictors of exercise capacity and everyday activity in older heart failure patients. European Journal of Heart Failure. 2006;8(2):203-207.

16. Patron E, Messerotti Benvenuti S, Lopriore V, Aratari J, Palomba D. Somatic-Affective, But Not Cognitive-Depressive Symptoms are Associated With Reduced Health-Related Quality of Life in Patients With Congestive Heart Failure. Psychosomatics. 2017;58(3):281-291. 
17. Chien $\mathrm{CL}$, Lee $\mathrm{CM}, \mathrm{Wu}$ YW, Wu YT. Home-based exercise improves the quality of life and physical function but not the psychological status of people with chronic heart failure: a randomised trial. Journal of Physiotherapy. 2011;57(3):157-163.

18. Uszko-Lencer N, Mesquita R, Janssen E, et al. Reliability, construct validity and determinants of 6minute walk test performance in patients with chronic heart failure. International Journal of Cardiology. 2017;240:285-290.

19. Evangelista LS, Cacciata M, Stromberg A, Dracup K. Dose-Response Relationship Between Exercise Intensity, Mood States, and Quality of Life in Patients With Heart Failure. The Journal of Cardiovascular Nursing. 2017;32(6):530-537.

20. Papasavvas T, Alhashemi M, Micklewright D. Association Between Depressive Symptoms and Exercise Capacity in Patients With Heart Disease: A META-ANALYSIS. Journal of Cardiopulmonary Rehabilitation and Prevention. 2017;37(4):239-249.

21. Conley S, Feder S, Redeker NS. The relationship between pain, fatigue, depression and functional performance in stable heart failure. Heart \& Lung . 2015;44(2):107-112.

22. Alosco ML, Spitznagel MB, Raz N, et al. The 2-minute step test is independently associated with cognitive function in older adults with heart failure. Aging Clinical and Experimental Research. 2012;24(5):468-474.

23. Moser DK, Arslanian-Engoren C, Biddle MJ, et al. Psychological Aspects of Heart Failure. Current Cardiology Reports. 2016;18(12):119.

24. Herr JK, Salyer J, Lyon DE, Goodloe L, Schubert C, Clement DG. Heart failure symptom relationships: a systematic review. The Journal of Cardiovascular Nursing. 2014;29(5):416-422.

25. Jaarsma T, Klompstra L, Ben Gal T, et al. Increasing exercise capacity and quality of life of patients with heart failure through Wii gaming: the rationale, design and methodology of the HFWii study; a multicentre randomized controlled trial. European Journal of Heart Failure. 2015;17(7):743-748.

26. Hamilton DM, Haennel RG. Validity and reliability of the 6-minute walk test in a cardiac rehabilitation population. Journal of Cardiopulmonary Rehabilitation. 2000;20(3):156-164.

27. Rostagno $C$, Olivo G, Comeglio $M$, et al. Prognostic value of 6 -minute walk corridor test in patients with mild to moderate heart failure: comparison with other methods of functional evaluation. European Jjournal of Heart Failure. 2003;5(3):247-252.

28. Costantini $M$, Musso $M$, Viterbori $P$, et al. Detecting psychological distress in cancer patients: validity of the Italian version of the Hospital Anxiety and Depression Scale. Supportive care in cancer 1999;7(3):121-127.

29. Bjelland I, Dahl AA, Haug TT, Neckelmann D. The validity of the Hospital Anxiety and Depression Scale. An updated literature review. Journal of Psychosomatic Research. 2002;52(2):69-77.

30. Haworth JE, Moniz-Cook E, Clark AL, Wang M, Cleland JG. An evaluation of two self-report screening measures for mood in an out-patient chronic heart failure population. International journal of Geriatric Psychiatry. 2007;22(11):1147-1153.

31. Nasreddine ZS, Phillips NA, Bedirian V, et al. The Montreal Cognitive Assessment, MoCA: a brief screening tool for mild cognitive impairment. Journal of the American Geriatrics Society. 2005;53(4):695-699.

32. Athilingam $P$, King KB, Burgin SW, Ackerman M, Cushman LA, Chen L. Montreal Cognitive Assessment and Mini-Mental Status Examination compared as cognitive screening tools in heart failure. Heart \& Lung : e. 2011;40(6):521-529.

33. Hawkins MA, Gathright EC, Gunstad J, et al. The MoCA and MMSE as screeners for cognitive impairment in a heart failure population: a study with comprehensive neuropsychological testing. Heart \& Lung. 2014;43(5):462-468. 
34. Keast ML, Slovinec D'Angelo ME, Nelson CR, et al. Randomized trial of Nordic walking in patients with moderate to severe heart failure. The Canadian Journal of Cardiology. 2013;29(11):14701476.

35. Gottlieb SS, Kop WJ, Ellis SJ, et al. Relation of depression to severity of illness in heart failure (from Heart Failure And a Controlled Trial Investigating Outcomes of Exercise Training [HFACTION]). The American Journal of Cardiology. 2009;103(9):1285-1289.

36. Freyssin C, Verkindt C, Prieur F, Benaich P, Maunier S, Blanc P. Cardiac rehabilitation in chronic heart failure: effect of an 8-week, high-intensity interval training versus continuous training. Archives of Physical Medicine and Rehabilitation. 2012;93(8):1359-1364.

37. Gary RA, Dunbar SB, Higgins MK, Musselman DL, Smith AL. Combined exercise and cognitive behavioral therapy improves outcomes in patients with heart failure. Journal of Psychosomatic Research. 2010;69(2):119-131.

38. Lesman-Leegte I, Jaarsma T, Sanderman R, Linssen G, van Veldhuisen DJ. Depressive symptoms are prominent among elderly hospitalised heart failure patients. European Journal of Heart Failure. 2006;8(6):634-640.

39. Klompstra L, Jaarsma T, Stromberg A. Physical activity in patients with heart failure: barriers and motivations with special focus on sex differences. Patient Preference and Adherence.

2015;9:1603-1610.

40. Polikandrioti M, Goudevenos J, Michalis LK, et al. Factors associated with depression and anxiety of hospitalized patients with heart failure. Hellenic journal of cardiology 2015;56(1):26-35.

41. Sokoreli I, de Vries JJ, Riistama JM, et al. Depression as an independent prognostic factor for allcause mortality after a hospital admission for worsening heart failure. International Journal of Cardiology. 2016;220:202-207.

42. Carson N, Leach L, Murphy KJ. A re-examination of Montreal Cognitive Assessment (MoCA) cutoff scores. International Journal of Geriatric Psychiatry. 2018;33(2):379-388.

43. Ciesielska N, Sokolowski R, Mazur E, Podhorecka M, Polak-Szabela A, Kedziora-Kornatowska K. Is the Montreal Cognitive Assessment (MoCA) test better suited than the Mini-Mental State Examination (MMSE) in mild cognitive impairment ( $\mathrm{MCl}$ ) detection among people aged over 60 ? Meta-analysis. Psychiatria polska. 2016;50(5):1039-1052.

44. Vellone E, Fida R, Ghezzi V, et al. Patterns of Self-care in Adults With Heart Failure and Their Associations With Sociodemographic and Clinical Characteristics, Quality of Life, and Hospitalizations: A Cluster Analysis. The Journal of Cardiovascular Nursing. 2017;32(2):180-189.

45. Cocchieri A, Riegel B, D'Agostino F, et al. Describing self-care in Italian adults with heart failure and identifying determinants of poor self-care. European Journal of Cardiovascular Nursing. 2015;14(2):126-136.

46. Athilingam P, Edwards JD, Valdes EG, Ji M, Guglin M. Computerized auditory cognitive training to improve cognition and functional outcomes in patients with heart failure: Results of a pilot study. Heart \& Lung. 2015;44(2):120-128.

47. Ghosh RK, Ball S, Prasad V, Gupta A. Depression in heart failure: Intricate relationship, pathophysiology and most updated evidence of interventions from recent clinical studies. International Journal of Cardiology. 2016;224:170-177.

48. Jeyanantham K, Kotecha D, Thanki D, Dekker R, Lane DA. Effects of cognitive behavioural therapy for depression in heart failure patients: a systematic review and meta-analysis. Heart Failure Reviews. 2017;22(6):731-741.

49. Lundgren JG, Dahlstrom O, Andersson G, Jaarsma T, Karner Kohler A, Johansson P. The Effect of Guided Web-Based Cognitive Behavioral Therapy on Patients With Depressive Symptoms and Heart Failure: A Pilot Randomized Controlled Trial. Journal of Medical Internet Research. 2016;18(8):e194. 
50. Viveiros J, Sethares K, Shapiro A. Repeated recall as an intervention to improve memory performance in heart failure patients. European Journal of Cardiovascular Nursing. 2017;16(8):724-732.

51. Norman J, Fu M, Ekman I, Bjorck L, Falk K. Effects of a mindfulness-based intervention on symptoms and signs in chronic heart failure: A feasibility study. European Journal of Cardiovascular Nursing. 2018;17(1):54-65.

52. Stott J, Spector A, Orrell M, Scior K, Sweeney J, Charlesworth G. Limited validity of the Hospital Anxiety and Depression Scale (HADS) in dementia: evidence from a confirmatory factor analysis. International Journal of Geriatric Psychiatry. 2017;32(7):805-813. 
Table 1: Sociodemographic and clinical characteristics of the sample $(n=96)$

\begin{tabular}{|c|c|}
\hline Characteristics & M (SD) or n (\%) \\
\hline Age $(M \pm S . D)$. & $72 \pm 10$ \\
\hline \multicolumn{2}{|l|}{ Male sex $(n, \%)$} \\
\hline Male & $70(73)$ \\
\hline \multicolumn{2}{|l|}{ Education (n, \%) } \\
\hline Primary School & $36(37)$ \\
\hline High School & $36(37)$ \\
\hline University or College & $23(24)$ \\
\hline Other & $1(1)$ \\
\hline \multicolumn{2}{|l|}{ Marital Status (n, \%) } \\
\hline Married/relationship & $56(58)$ \\
\hline Single/divorced/widowed & 37 (39) \\
\hline Other (divorced, widowed) & $3(3)$ \\
\hline \multicolumn{2}{|l|}{ Smoking $(n, \%)$} \\
\hline Yes & $20(21)$ \\
\hline \multicolumn{2}{|l|}{ Etiology of HF (n, \%) } \\
\hline Ischemia & $19(20)$ \\
\hline Hypertension & $55(57)$ \\
\hline Cardiomyopathy & $61(63)$ \\
\hline Valvular heart disease & 27 (28) \\
\hline \multicolumn{2}{|l|}{ ECG Rhythm (n, \%) } \\
\hline Sinus & $64(67)$ \\
\hline Atrial fibrillation & $19(20)$ \\
\hline Pacemaker & $12(12)$ \\
\hline Unknown & $1(1)$ \\
\hline Ejection fraction $(M \pm S . D)$. & $44 \pm 10$ \\
\hline \multicolumn{2}{|l|}{ NYHA class (n, \%) } \\
\hline I & $4(4)$ \\
\hline II & $48(50)$ \\
\hline III & $41(43)$ \\
\hline IV & $3(3)$ \\
\hline Body Mass Index & $29,95 \pm 32,30$ \\
\hline Time since diagnosis (months) $(M \pm S . D$.) & $52 \pm 80$ \\
\hline \multicolumn{2}{|l|}{ Medications (n, \%) } \\
\hline Beta Blockers & $52(54)$ \\
\hline Angiotensin Receptor Blocking agents & $13(13)$ \\
\hline Angiotensin-Converting Enzyme inhibitors & $27(28)$ \\
\hline Diuretics & $85(88)$ \\
\hline \multicolumn{2}{|l|}{ Exercise during the last week ( $n, \%)$} \\
\hline None & $13(14)$ \\
\hline$<30 \mathrm{~min} /$ week & $5(5)$ \\
\hline 30-60 min/week & $7(7)$ \\
\hline 1-3 hours/week & $8(8)$ \\
\hline >3 hours/week & $63(66)$ \\
\hline
\end{tabular}


Table 2. Correlation matrix of the studied variables $(n=96)$

\begin{tabular}{|c|c|c|c|c|c|c|c|c|c|}
\hline & 6MWT & HADS-A & HADS-D & MoCA & Age & Gender & Education & NYHA class & BMI \\
\hline 6MWT & 1 & & & & & & & & \\
\hline HADS-A & $-.231^{*}$ & 1 & & & & & & & \\
\hline HADS-D & $-.325^{* *}$ & $.644^{* *}$ & 1 & & & & & & \\
\hline MoCA & $.244^{*}$ & .031 & -.092 & 1 & & & & & \\
\hline Age &.$- .451^{* *}$ & -.013 & .141 & $-.528^{* *}$ & 1 & & & & \\
\hline Gender &.$- .407^{* *}$ & .054 & .146 & -.135 & .197 & 1 & & & \\
\hline Education & .115 & -.041 & .038 & $.242^{*}$ & $-.304^{* *}$ & .152 & 1 & & \\
\hline NYHA class & $-.540^{* *}$ & $.248^{*}$ & $.223^{*}$ & -.174 & $.341^{* *}$ & $.241^{*}$ & -.147 & 1 & \\
\hline BMI & .001 & -.110 & .098 & -.042 & -.074 & -.009 & $-.272^{* *}$ & $.271^{* *}$ & 1 \\
\hline
\end{tabular}

${ }^{*} \mathrm{p}<.05 ;{ }^{* *} \mathrm{p}<.001$

${ }^{\$}$ variable dichotomized: $0=$ male; $1=$ female

Abbreviations: 6MWT= 6-min walk test; HADS-A= Hospital Anxiety and Depression Scale - Anxiety score; HADS-D= Hospital Anxiety and Depression Scale - Depression score; MoCA= Montreal Cognitive Assessment; NYHA= New York Heart Association; BMI= Body Mass Index 
Figure 1: Meters walked at 6MWT - Differences among MoCA categories (n=96)

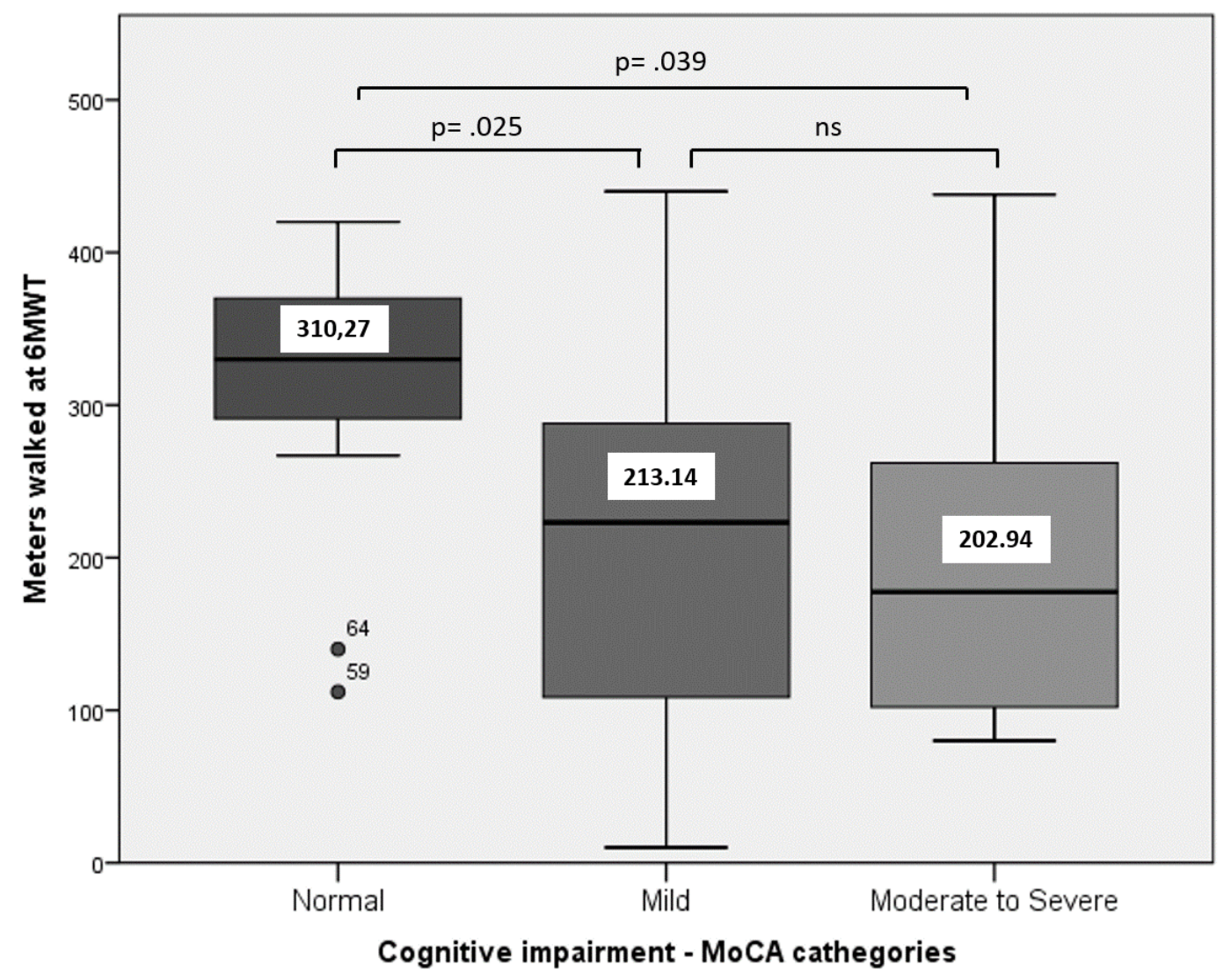


Table 3: Predictors of exercise capacity $(\mathrm{N}=96)$

\begin{tabular}{|c|c|c|c|c|c|}
\hline Variables & Standardized $\beta$ & $\mathrm{p}$ & VIF & $\mathrm{R}^{2}$ & $\mathrm{~F}$ \\
\hline \multicolumn{6}{|l|}{ Predictors of exercise capacity } \\
\hline NYHA class & -.329 & .000 & 1.2 & & \\
\hline Age & -.291 & .001 & 1.1 & & \\
\hline Gender ( $0=$ male; $1=$ female) & -.248 & .002 & 1.1 & & \\
\hline \multirow[t]{2}{*}{ Depression (HADS) } & -.188 & .019 & 1.1 & & \\
\hline & & & & 0.474 & $20.49 * *$ \\
\hline
\end{tabular}

HADS= Hospital Anxiety and Depression Scale; NYHA= New York Heart Association 